\title{
Tobacco-specific carcinogen nitrosamine 4-(methylnitrosamino)-1-(3-pyridyl)-1-butanone induces AKT activation in head and neck epithelia
}

\author{
STEPHEN M. WEBER ${ }^{1}$, SOPHIA BORNSTEIN ${ }^{1}$, YUEXIN LI ${ }^{1}$, STEPHEN P. MALKOSKI ${ }^{2}$, \\ DONNA WANG ${ }^{3}$, ANIL K. RUSTGI ${ }^{4}$, MOLLY F. KULESZ-MARTIN ${ }^{5}$, XIAO-JING WANG ${ }^{3}$ and SHI-LONG LU ${ }^{6}$ \\ ${ }^{1}$ Department of Otolaryngology, Oregon Health \& Science University, Portland, OR; \\ Departments of ${ }^{2}$ Pulmonary and Critical Care Medicine and ${ }^{3}$ Pathology, University of Colorado Denver, \\ Aurora, $\mathrm{CO} ;{ }^{4}$ Department of Medicine (Division of Gastroenterology) and Genetics, Abramson Cancer Center, \\ University of Pennsylvania, Philadelphia, PA; ${ }^{5}$ Department of Dermatology, Oregon Health \& Science University, \\ Portland, OR; ${ }^{6}$ Departments of Otolaryngology, University of Colorado Denver, Aurora, CO, USA
}

Received May 6, 2011; Accepted June 14, 2011

DOI: $10.3892 /$ ijo.2011.1149

\begin{abstract}
Exposure to tobacco carcinogens is causally associated with head and neck squamous cell carcinoma (HNSCC), but the underlying molecular mechanisms remain unclear. Here, we reported that AKT is activated at a higher frequency in both HNSCC tumors and the adjacent mucosa from HNSCC patients who are smokers than those from HNSCC patients who are non-smokers. Adding physiologically relevant concentrations of 4-(methylnitrosamino)-1-(3-pyridyl)-11butanone (NNK), a major tobacco carcinogen, to normal head and neck epithelial cells and HNSCC cell lines, rapidly and constitutively activated AKT through phosphorylation in a dose- and time-dependent manner. AKT phosphorylation was associated with activation of downstream signaling mediators BAD, MDM2, GSK-3 $\beta$, mTOR. These alterations correlated with increased proliferation and decreased etoposide-induced apoptosis in NNK-exposed cells. Finally, NNK exposure to mouse head and neck epithelia resulted in epithelial hyperproliferation and reduced apoptosis, which is correlated with AKT activation. Our results suggest that AKT activation is an early event and plays a pivotal role in mediating tobaccoinduced HNSCC carcinogenesis.
\end{abstract}

Correspondence to: Dr Shi-Long Lu, Department of Otolaryngology, University of Colorado Denver, RC-2, P15-7112, 12700 E 19th Avenue, Aurora, CO 80045, USA

E-mail: shi-long.lu@ucdenver.edu

Abbreviations: HNSCC, head and neck squamous cell carcinoma; NNK, nitrosamine 4-(methylnitrosamino)-1-(3-pyridyl)-1-butanone; PI3K, phosphatidylinositol-3-kinase; PTEN, phosphatase and tensin homologue; IHC, immunohistochemistry; TUNEL, Tdt-mediated dUTP nick-end labeling; BrdU, bromodeoxyuridine

Key words: tobacco, NNK, AKT, head and neck epithelia, head and neck squamous cell carcinoma

\section{Introduction}

Tobacco use is the leading cause of preventable cancer worldwide and accounts for at least $30 \%$ of cancer deaths (1).Epidemiological studies have clearly documented a strong causal link between tobacco use and an increased risk for head and neck squamous cell carcinoma (HNSCC) (2), however, the underlying molecular mechanism is still poorly understood. Patients with HNSCC who smoke often present with multiple precancerous and cancerous lesions, so called 'field cancerization' throughout the upper aerodigestive tract, where the head and neck mucosa is chronically exposed to tobacco carcinogens (3). Tobacco-induced carcinogenesis is a multiple-step process requiring accumulation of numerous genetic and epigenetic alterations throughout the exposed tissue. One major tobacco carcinogen, 4-(methylnitrosamino)-1-(3-pyridyl)-1-1butanone (NNK), is a nitrosamine formed during curing and processing of nicotine and other tobacco alkaloids. It is well established that NNK directly damages DNA through the formation of covalently bound DNA adducts (1). Persistence of DNA adducts that have escaped cellular repair can result in permanent mutations in a tumor suppressor gene or an oncogene. These effects on cellular DNA are generally referred to as the 'genotoxic effects' of tobacco carcinogen (1).

Recent studies have shown that NNK also directly activates multiple signal transduction pathways (1). For example, NNK activates AKT, a serine/threonine kinase that regulates cellular survival in normal and cancer cells in the lung, although the mechanism remains unclear $(4,5)$. AKT is a major downstream effecter in the phosphatidylinositol-3-kinase (PI3K) pathway, and is negatively regulated by the tumor suppressor, phosphatase and tensin homologue (PTEN). Activated AKT regulates its multiple downstream targets to decrease apoptosis and increase proliferation, all of which will synergistically increase the direct genotoxic effects of NNK to promote carcinogenesis (6).

Unlike lung cancers which are primarily adenocarcinoma, HNSCC are derived from stratified keratinocytes. Therefore, molecular mechanisms of HNSCC are distinct from lung 
cancers. For instance, K-ras mutation, which frequently occurs in lung cancer, occurs at a much lower rate in HNSCC, whereas overexpression of K-ras at transcriptional level is common in HNSCC (7). While the genotoxic effects of tobacco carcinogens in HNSCC carcinogenesis have been described (8), no studies have explored the signaling pathways related to tobacco-induced HNSCC carcinogenesis. To investigate the molecular mechanism of tobacco-induced HNSCC carcinogenesis, in the present study, we first examined AKT activation in human HNSCC tumors and their adjacent mucosa from smokers and nonsmokers. We then exposed NNK, at concentrations achievable by smokers, to normal and head and neck cancer cells to study the status of AKT activation. Finally, we applied NNK to oral cavity of normal mice to observe the NNK-induced AKT activation in vivo. Our current study suggests the activation of AKT is one of the molecular mechanisms in mediating tobacco-induced HNSCC carcinogenesis.

\section{Patients and methods}

Patients. Under an Institutional Review Board approved protocol and following informed consent, HNSCC and case-matched adjacent mucosa tissues from 35 surgical specimens resected from 2000 to 2005 at the Department of Otolaryngology, Oregon Health \& Science University, were collected. Patient records were retrospectively reviewed for smoking exposure as assessed by trained physicians at diagnosis. A patient was considered as a smoker if he admitted any active smoking history. Patients who denied any active smoking exposure were defined as nonsmokers. Among the tissues assayed were 15 tongue SCCs, 9 oral SCCs, 4 pharyngeal SCCs, 7 larynx SCCs and case-matched adjacent uninvolved tissue. An additional seven normal control tissue samples were obtained from healthy adults undergoing surgery for obstructive sleep apnea. All tumor samples were histologically analyzed to confirm the presence of $80 \%$ carcinoma cells and absence of large necrotic or stromal areas.

Cell lines. Human normal oral epithelial cells OKF6 and normal esophageal squamous epithelial cells EPC2 were cultured as described previously (9). Human HNSCC cell lines SCC9, SCC4, and SCC25 were purchased from the American Type Culture Collection (Manassas, VA) and maintained under standard conditions in the suppliers recommend growth medium.

Western blotting. Western blotting was performed as described previously (7) using an antibody against total AKT, phosphorylation-specific antibodies against p-AKT (Ser473, Thr308), p-GSK3 $\alpha / \beta$ (Ser21/9), p-mTOR (Ser2448), p-BAD (Ser136), p-MDM2 (Ser166) (Cell Signaling, Danvers, MA), and detected using the Odyssey infrared detection system (Li-COR Biosciences, Lincoln, NE).

Cell proliferation and apoptosis. Cell proliferation was measured using a colorimetric MTT (3-(4,5-Dimethylthiazol-2-yl)-2, 5-diphenytetrazolium bromide) assay according to the manufaturer's instructions (Chemicon International, Temecula, CA). To induce apoptosis, OKF6 cells were treated with etoposide (Calbiochem, Gibbstown, NJ) in the presence or absence of NNK (Toronto Research Chemicals, North York, ON, Canada). The apoptotic rate was determined using the annexin V-FITC apoptosis detection kit (BioVision, Mountain View, CA) and quantitated by flow cytometry.

Animal studies. Animals were housed at the Oregon Health \& Science University animal facility under standard conditions with a 12-h day-night cycle. All protocols were approved by the Institutional Animal Care and Use Committee. NNK $(100 \mu \mathrm{M})$ in $100 \mu \mathrm{l}$ sesame oil or sesame oil vehicle was applied to the oral mucosa of 8-weeks old C57BL/6 mice three times/week for 4 weeks. Animals were then euthanized and head and neck tissues were harvested. For proliferation studies, bromodeoxyuridine (BrdU, $0.125 \mathrm{mg} / \mathrm{g}$, i.p.) was administered $1 \mathrm{~h}$ prior to euthanization.

Immunostaining. Immunohistochemistry (IHC) was performed on paraffin-embedded sections as previously reported (10) using rabbit polyclonal phosphor-AKT IHC-specific antibody. IHC results for phosphorylated AKT were reviewed by two independent researchers who were blinded for tobacco use, and were deemed positive for AKT activation if $>30 \%$ of the cells stained phospho-AKT positive (Ser473) per field of view. Immunoflurorescence of BrdU labeling was performed using FITC-conjugated anti-BrdU antibody (BD Biosciences, San Jose, CA), and counterstained by keratin 14 antibody (Fitzgerald, Concord, MA). Immunofluorescence of Tdt-mediated dUTP nick-end labeling (TUNEL) assays was performed using the TUNEL assay kit (Promega, Madison, WI), and counterstained with propidium iodine.

\section{Results and Discussion}

AKT activation has been reported in several types of cancer, including HNSCC (11). To evaluate whether AKT activation correlates with tobacco exposure in HNSCC, we examined smoking histories of HNSCC patients and the status of AKT activation. A total of 35 pairs of HNSCC samples and casematched adjacent mucosa samples were analyzed. Seven normal oropharyngeal samples from sleep apnea patients were used as normal controls. Among 35 pairs of tissue samples examined, 23 pairs were from HNSCC patients who are smokers and 12 pairs are from HNSCC patients who are non-smokers as defined in Materials and methods. Immunohistochemistry (IHC) using a phosphorylation-specific antibody revealed that AKT activation was detected more frequently in both the adjacent mucosa (61 vs. 17\%, p<0.05) and HNSCC arising from smokers (91 vs. 58\%, p<0.05) than those from HNSCC patients who are non-smokers (Fig. 1). None of the normal controls exhibited positive staining for activated AKT (Fig. 1). We cannot exclude the possibility that the lower percentage of AKT activation detected in the adjacent mucosa and HNSCC tumors of non-smokers is due to second-hand smoking or exposure to other environmental carcinogens. Baseline activation in HNSCC tumors is not surprising, since AKT activation is common in multiple cancer types (12).

In order to explore whether the correlation between tobacco smoking and AKT activation represent a causal role of tobacco carcinogens in AKT activation, we exposed human normal oral epithelial cells (OKF6), normal esophageal squamous epithelial cells (EPC2) and HNSCC cell lines (SCC9, SCC4, and SCC25) to NNK. As shown in Fig. 2, NNK rapidly induced phos- 
A

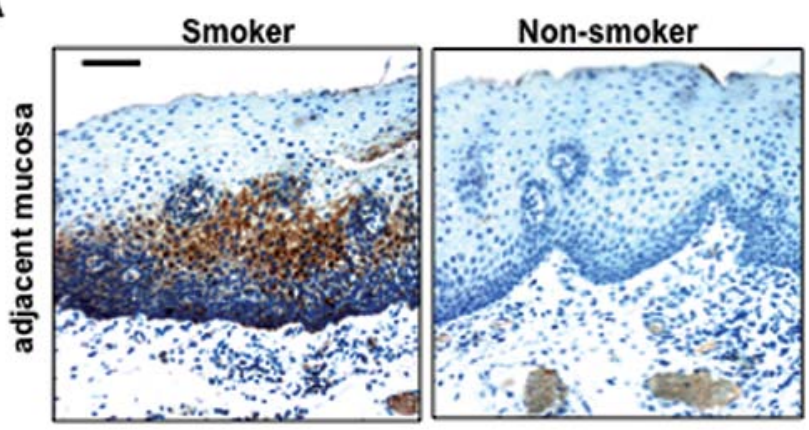

B

\begin{tabular}{|c|c|}
\hline Sample type (total \#) & AKT activation \\
\hline Normal control (7) & $0(0 \%)$ \\
\hline $\begin{array}{c}\text { Non-smoker mucosa } \\
(12)\end{array}$ & $2(17 \%)$ \\
\hline $\begin{array}{c}\text { Non-smoker HNSCC } \\
(12)\end{array}$ & $7(58 \%)^{a . b}$ \\
\hline Smoker mucosa (23) & $14(61 \%)^{\text {a.b }}$ \\
\hline Smoker HNSCC (23) & $21(91 \%)^{3 . b . c . d}$ \\
\hline
\end{tabular}

Figure 1. AKT activation occurs more frequently in the adjacent mucosa and HNSCC tumors from smokers than non-smokers. (A) Representative IHC sections of adjacent mucosa obtained from a smoker (left panel) and nonsmoker (right panel) stained for active AKT (phosphorylated at Ser473). The bar in the first panel represents $40 \mu \mathrm{m}$. (B) Summary of AKT activation in human HNSCC samples. The $\chi^{2}$ test $(\mathrm{p}<0.05)$ was used for comparisons with (a) normal controls, (b) non-smoker adjacent mucosa, (c) non-smoker HNSCC, (d) smoker adjacent mucosa. phorylation of AKT at both Ser473 and Thr308 in a dose- and time-dependent manner in all cell lines. AKT phosphorylation at both sites was evident as early as $15 \mathrm{~min}$ after $10^{-8} \mathrm{M}$ NNK exposure, reached to a maximum level between the 30- and 60-min time-points (Fig. 2, left panel), and the increased phosphorylation status was maintained for $24 \mathrm{~h}$ (Fig. 3A). To determine dose-dependent response to NNK, AKT was activated in each cell type with NNK doses as low as $10^{-8}-10^{-7} \mathrm{M}$, but maximum phosphorylation was observed at $10^{-4} \mathrm{M}$ (Fig. 2, right panel). These concentrations are physiologically relevant, as average steady-state serum concentration of NNK in active smokers has been reported at $2 \times 10^{-7} \mathrm{M}$, and acute increases to $10^{-4}-10^{-5} \mathrm{M}$ in serum or to $10^{-3} \mathrm{M}$ at the mucosal surface immediately after smoking have also been reported (13-15).

To determine whether AKT phosphorylation triggered downstream signaling within normal head and neck epithelial cells, we assessed the phosphorylation status of several known AKT downstream signaling mediators, Bad, Mdm2, GSK3 $\alpha / \beta$ and mTOR, which play a role in regulating apoptosis and/or proliferation. Bad is inactivated through phosphorylation, which in turn frees the survival factor BCL-X $\mathrm{X}_{\mathrm{L}}$ to exert its anti-apoptotic effects (16). Phosphorylation of $\mathrm{Mdm} 2$ results in increased p53 degradation, which blunts the apoptotic-inducing role of p53 (17). In addition, AKT activation also phosphorylates and inactivates GSK3 $\beta$, which can prevent its degradation of cyclin D1 and c-myc by GSK3 $\beta$ phosphorylation. Finally, the mTOR pathway is well known for protein translational control and cell growth, and is a direct downstream target of AKT by phosphorylation. We observed increased phosphorylation of each of these downstream targets following NNK exposure (Fig. 3). To assess the requirement of AKT activation for NNK-induced
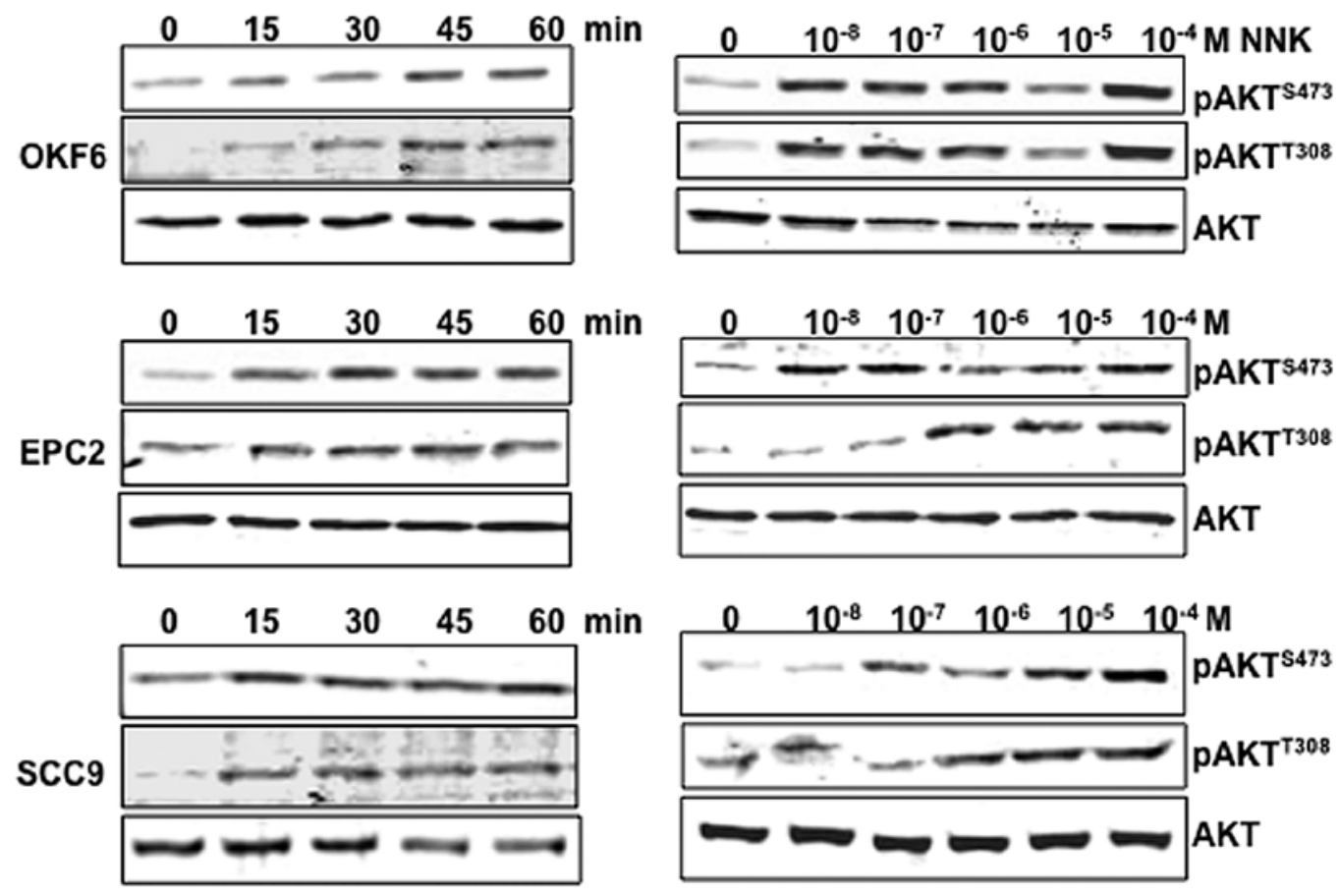

Figure 2. AKT activation upon NNK exposure in normal head and neck epithelial cells and an HNSCC cell line. Left panels, time-dependent induction of AKT phosphorylation by NNK. Cells were plated and cultured in 6-well plates. The next day, the medium was changed to DMEM with or without NNK (10 $\left.{ }^{-8} \mathrm{M}\right)$ and cells were incubated for the indicated time. Right panels, dose-dependent induction of AKT phosphorylation by NNK. Cells were processed as described above and were exposed to the indicated doses of NNK for $1 \mathrm{~h}$. Cells were harvested and processed for Western blotting using phospho-specific antibodies against Ser473 or Thr308 and antibody against total AKT. 
A

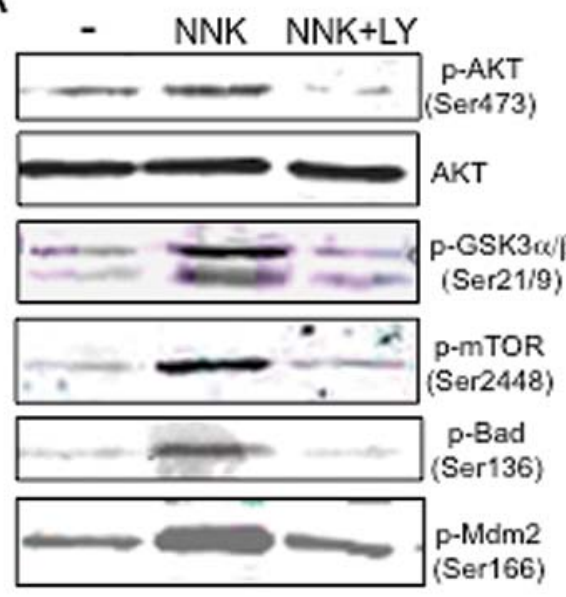

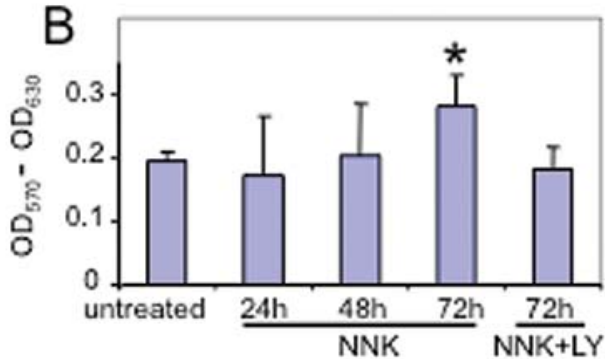

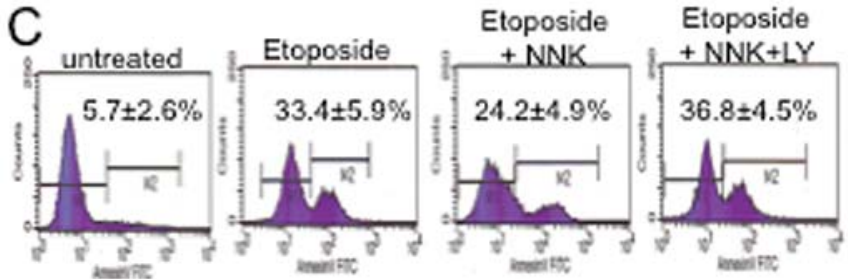

Figure 3. Effects of AKT activation on downstream targets, cell proliferation, and apoptosis. (A) Effects of AKT activity on downstream targets. OFK6 cells were exposed to $10^{-8} \mathrm{M}$ NNK with and without addition of $10 \mu \mathrm{M} \mathrm{LY} 294002$ for $24 \mathrm{~h}$. Cells were harvested and processed for Western blotting using the indicated phospho-specific antibodies and an antibody against total AKT. (B) Effects of NNK exposure on OKF6 cell proliferation. Cells were plated in 96-well plates, and were exposed to $10^{-8} \mathrm{M}$ NNK for 24, 48 and $72 \mathrm{~h}$ with or without $10 \mu \mathrm{M} \mathrm{LY} 294002(\mathrm{LY})$. The cells were then added to MTT solution, and the absorbance was measured at 570 and $630 \mathrm{~nm}$ (reference) using a microplate reader. Values are averaged from triplicates. " $\mathrm{p}<0.05$ (Student's t-test) in comparison with untreated cells. (C) Effects of NNK on OKF6 cell apoptosis. OKF cells were plated in 6-well plates in triplicates and were treated with $20 \mu \mathrm{M}$ etoposide for $24 \mathrm{~h}$ with or without addition of $10^{-8} \mathrm{M}$ NNK. The data shown in the far right panel represent cells treated with etoposide and $10 \mu \mathrm{M} \mathrm{LY} 294002$ for $24 \mathrm{~h}$. The apoptosis rate was determined using Annexin V-FITC apoptosis detection kit and quantified by flow cytometry. M2 represents the apoptotic cell population. Values were average from triplicates with standard deviation.
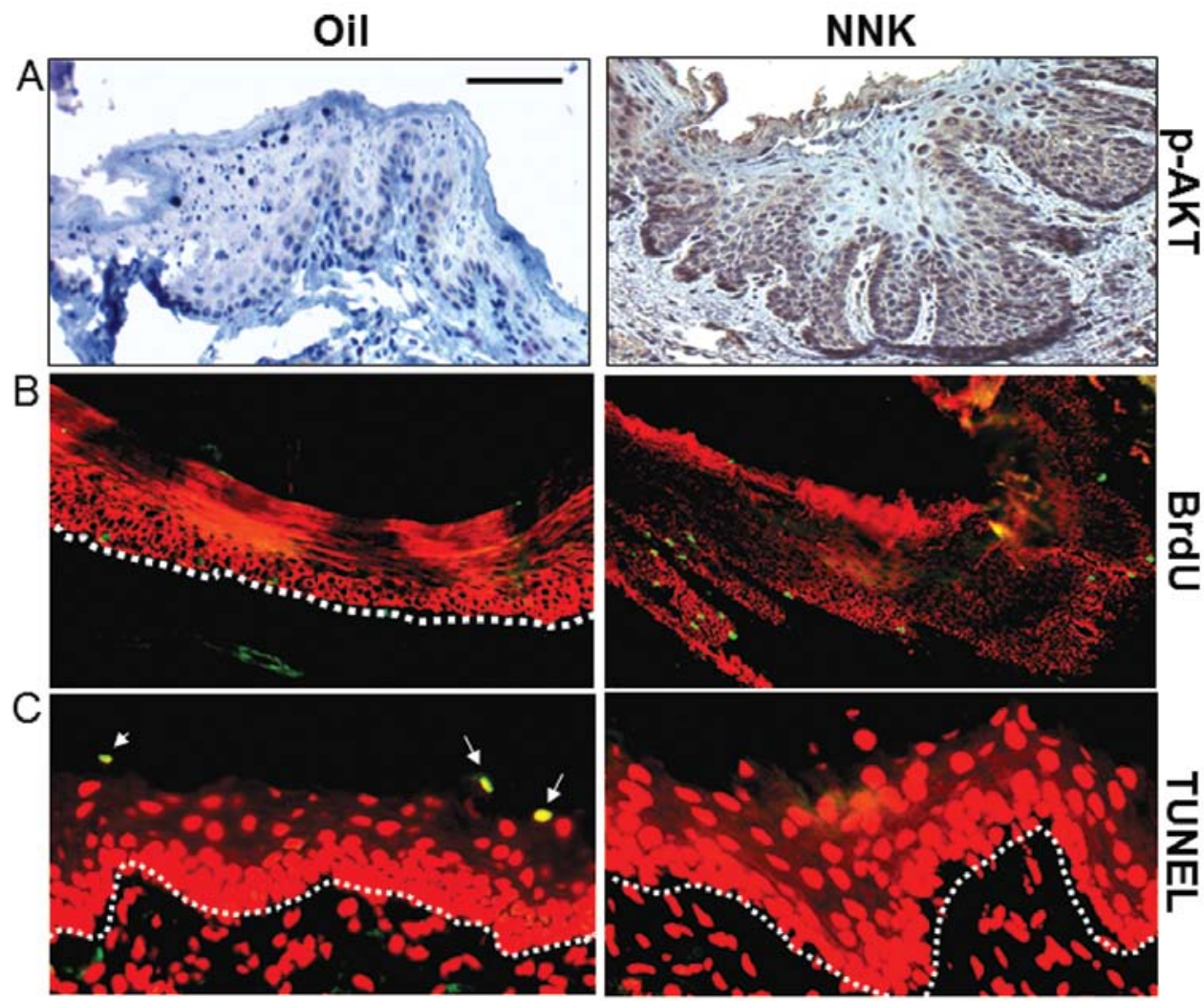

Figure 4. Mice exposed to NNK exhibit AKT activation, increased cell proliferation, and epithelial hyperplasia with no head and neck tumor formation. (A) NNK exposure induced AKT activation. Buccal mucosa, were harvested from 10-week old C57B6 mice exposed orally to $100 \mu \mathrm{M}$ NNK/mouse 3 times per week for 4 weeks. IHC staining was performed using the phosphorylation specific AKTSer473 antibody. (B) NNK exposure increases cell proliferation. Mice were injected with $\mathrm{BrdU}(0.125 \mathrm{mg} / \mathrm{g}) 1 \mathrm{~h}$ before harvesting head and neck tissues. Immunofluorescence staining to detect BrdU revealed increased proliferation in epithelial cells (green) of NNK-exposed buccal mucosa in comparison with control (oil-exposed) tissues. The epithelial compartment in the tissues is highlighted using a K14 (red) antibody. (C) NNK exposure decreases cell apoptosis. A TUNEL assay (Promega) revealed obvious apoptotic cells (yellow) in the buccal mucosa from control mice (left panel), whereas almost no apoptotic cells were detected in the same tissues from mice exposed to NNK (right panel). The countertstain in red is propidium iodide (PI). The dotted lines delineate the epithelium-stromal boundary. The bar in the first panel represents $30 \mu \mathrm{m}$ in (A), $40 \mu \mathrm{m}$ in (B), and $20 \mu \mathrm{m}$ in (C). 
phosphorylation of these downstream mediators, we pre-treated cells with a PI3K/AKT inhibitor, LY294002. As shown in Fig. 3A, LY294002 pre-treatment significantly blocked NNK-induced AKT phosphorylation at Ser473 without altering total AKT levels, thus LY294002 is able to block AKT activation. Furthermore, blocking AKT activity resulted in decreased phosphorylation of these key downstream mediators, indicating NNK-induced activation of these molecules depends on activated AKT (Fig. 3A).

To determine whether NNK-induced AKT activation and subsequent downstream molecular changes are able to affect cell growth and survival, we analyzed cell proliferation using a colorimetric MTT assay. As shown in Fig. 3B, when OKF6 cells were exposed to NNK, cell proliferation was significantly higher after $72 \mathrm{~h}$ of NNK exposure in comparison with non-NNK exposed OKF6 cells. Further, this effect was completely blocked by LY294002 treatment confirming the requirement for PI3K/ AKT signaling. We then assessed the effect of NNK-induced AKT activation on cell apoptosis. OKF6 cells were treated using a topoisomerase II inhibitor, etoposide, to induce cell apoptosis with or without NNK exposure. As shown in Fig. 3C, in cells that were not exposed to NNK, etoposide increased cell apoptosis from 5.7 to $33.4 \%$, as measured by annexin V-FITC staining. In the presence of NNK, etoposide-induced apoptosis was significantly decreased to $24.2 \%(\mathrm{p}<0.05)$. Furthermore, this NNK-induced attenuation of apoptosis was completely abolished by LY294002 treatment (36.8\%).

To investigate whether NNK exposure activates AKT in vivo, C57BL/6 mice were orally treated with $100 \mu \mathrm{M}$ NNK dissolved in sesame oil three times per week. Four weeks of NNK exposure produced a hyperplastic and thickened epithelium. AKT activation was detected in murine buccal mucosa (Fig. 4A), tongue and esophagus (data not shown) following exposure to NNK but not sesame oil control. To determine whether epithelial hyperplasia was, at least in part, due to cell proliferation and/ or increased survival, a bromodeoxyuridine (BrdU) labeling experiment was performed. NNK exposure resulted in 5.3-fold increase in BrdU labeling index in mouse head and neck tissues (17.5 \pm 2.4 nuclei $/ \mathrm{mm}$ basement membrane, $n=5$, Fig. $4 \mathrm{~B})$ in comparison with oil-exposed control mice (3.3 \pm 1.2 nuclei/ $\mathrm{mm}$ basement membrane, n=5, Fig. 4B). Additionally, terminal deoxynucleotidyltransferase-dUTP nick-end labeling (TUNEL) assay revealed a nearly complete blockade of apoptosis in head and neck tissues from mice with NNK exposure as compared with control tissues (Fig. 4C).

Given what has been previously demonstrated with regard to the 'genotoxic' effects of tobacco carcinogens, our data showing NNK-induced AKT activation in head and neck epithelium and HNSCC provide a novel mechanism of tobacco-induced HNSCC carcinogenesis. The decreased apoptosis and increased proliferation of cell damaged by NNK will synergistically augment the 'genotoxic' effects of NNK, which would greatly enhance the potency of tobacco carcinogens. Thus, our data indicate that AKT activation may resent a key molecular pathway involved in tobacco-induced carcinogenesis.

AKT activation is observed in adjacent mucosa of smokers at nearly four-times more than in non-smokers, suggesting that AKT activation by tobacco carcinogen occurs early in the development of HNSCC. Thus, AKT activation upon tobacco exposure could be a potential target for chemopreventive approach of HNSCC. In fact, there are several recent reports that deguelin, a naturally occurring rotenoid, possesses chemopreventative role on lung cancer through inhibiting PI3K-AKT signaling $(18,19)$. In addition, HNSCC patients have a $10 \%$ risk of developing a second primary malignancy within 5 years after primary surgical treatment (20) and these patients require close follow-up for recurrence. Tobacco use is an important risk factor for the HNSCC recurrence (20). Thus, AKT activation upon tobacco exposure could have a dramatic impact on the HNSCC recurrence. Therefore, further investigation of various inhibitors of PI3K/AKT pathway in both in vitro and in animal models of HNSCC will provide valuable information for developing new strategy of chemoprevention and/or treatment for HNSCC.

In conclusion, our studies show that AKT activation is an early event in human HNSCC development, and is associated with smoking history. In addition, application of physiologically relevant concentrations of NNK to normal head and neck epithelial and HNSCC cells activates AKT, with subsequently increased cell proliferation and survival. Furthermore, NNK exposure in vivo results in AKT activation in mouse head and neck epithelia. Thus, our studies here represent the first report that AKT activation upon NNK exposure is one of the molecular mechanisms of tobacco-induced HNSCC carcinogenesis.

\section{Acknowledgements}

This work was partially supported by the THANC (Thyroid, Head and Neck Cancer) Foundation Grant, and Dermatology Foundation Career Development Grant to S.L. Lu. S.M. Weber is a recipient for the American Academy of Otolaryngology-Head and Neck Surgery Foundation (AAO-HNSF) Resident Research Award. A.K. Rustgi is supported by NCI P01 CA 098101.

\section{References}

1. Hecht SS: Tobacco carcinogens, their biomarkers and tobaccoinduced cancer. Nat Rev Cancer 3: 733-744, 2003.

2. Vineis P, Alavanja M, Buffler P, et al: Tobacco and cancer: recent epidemiological evidence. J Natl Cancer Inst 96: 99-106, 2004.

3. Mao L, Hong WK and Papadimitrakopoulou VA: Focus on head and neck cancer. Cancer Cell 5: 311-316, 2004.

4. West KA, Brognard J, Clark AS, et al: Rapid Akt activation by nicotine and a tobacco carcinogen modulates the phenotype of normal human airway epithelial cells. J Clin Invest 111: 81-90, 2003.

5. West KA, Linnoila IR, Belinsky SA, Harris CC and Dennis PA: Tobacco carcinogen-induced cellular transformation increases activation of the phosphatidylinositol 3'-kinase/Akt pathway in vitro and in vivo. Cancer Res 64: 446-451, 2004.

6. Vivanco I and Sawyers CL: The phosphatidylinositol 3-kinase AKT pathway in human cancer. Nat Rev Cancer 2: 489-501, 2002.

7. Lu SL, Herrington H, Reh D, et al: Loss of transforming growth factor-beta type II receptor promotes metastatic head-and-neck squamous cell carcinoma. Genes Dev 20: 1331-1342, 2006.

8. Brennan JA, Boyle JO, Koch WM, et al: Association between cigarette smoking and mutation of the p53 gene in squamous-cell carcinoma of the head and neck. N Engl J Med 332: 712-717, 1995.

9. Andl CD, Mizushima T, Nakagawa H, et al: Epidermal growth factor receptor mediates increased cell proliferation, migration, and aggregation in esophageal keratinocytes in vitro and in vivo. J Biol Chem 278: 1824-1830, 2003.

10. Lu SL, Reh D, Li AG, et al: Overexpression of transforming growth factor betal in head and neck epithelia results in inflammation, angiogenesis, and epithelial hyperproliferation. Cancer Res 64: 4405-4410, 2004 
11. Amornphimoltham P, Sriuranpong V, Patel V, et al: Persistent activation of the Akt pathway in head and neck squamous cell carcinoma: a potential target for UCN-01. Clin Cancer Res 10: 4029-4037, 2004

12. Altomare DA and Testa JR: Perturbations of the AKT signaling pathway in human cancer. Oncogene 24: 7455-7464, 2005.

13. Russell MA, Jarvis M, Iyer R and Feyerabend C: Relation of nicotine yield of cigarettes to blood nicotine concentrations in smokers. Br Med J 280: 972-976, 1980.

14. Armitage AK, Dollery CT, George CF, Houseman TH, Lewis PJ and Turner DM: Absorption and metabolism of nicotine from cigarettes. Br Med J 4: 313-316, 1975.

15. Lindell G, Farnebo LO, Chen D, et al: Acute effects of smoking during modified sham feeding in duodenal ulcer patients. An analysis of nicotine, acid secretion, gastrin, catecholamines, epidermal growth factor, prostaglandin E2, and bile acids. Scand J Gastroenterol 28: 487-494, 1993.
16. Datta SR, Dudek H, Tao X, et al: Akt phosphorylation of BAD couples survival signals to the cell-intrinsic death machinery. Cell 91: 231-241, 1997.

17. Mayo LD and Donner DB: A phosphatidylinositol 3-kinase/Akt pathway promotes translocation of Mdm2 from the cytoplasm to the nucleus. Proc Natl Acad Sci USA 98: 11598-11603, 2001.

18. Chun KH, Kosmeder JW II, Sun S, et al: Effects of deguelin on the phosphatidylinositol 3-kinase/Akt pathway and apoptosis in premalignant human bronchial epithelial cells. J Natl Cancer Inst 95: 291-302, 2003.

19. Lee HY, Oh SH, Woo JK, et al: Chemopreventive effects of deguelin, a novel Akt inhibitor, on tobacco-induced lung tumorigenesis. J Natl Cancer Inst 97: 1695-1699, 2005.

20. Lin K, Patel SG, Chu PY, et al: Second primary malignancy of the aerodigestive tract in patients treated for cancer of the oral cavity and larynx. Head Neck 27: 1042-1048, 2005. 